\title{
A study of the almost sequential mechanism of true ternary fission
}

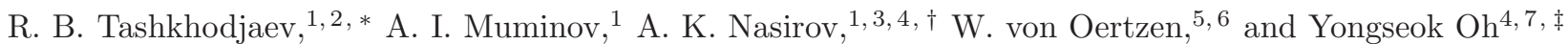 \\ ${ }^{1}$ Institute of Nuclear Physics, Uzbek Academy of Science, 100214 Tashkent, Uzbekistan \\ ${ }^{2}$ Inha University in Tashkent, 100170, Tashkent, Uzbekistan \\ ${ }^{3}$ Joint Institute for Nuclear Research, Joliot-Curie 6, 141980 Dubna, Russia \\ ${ }^{4}$ Department of Physics, Kyungpook National University, Daegu 702-701, Korea \\ ${ }^{5}$ Helmholtz-Zentrum Berlin, Glienickerstr. 100, 14109 Berlin, Germany \\ ${ }^{6}$ Fachbereich Physik, Freie Universität, Berlin, Germany \\ ${ }^{7}$ Asia Pacific Center for Theoretical Physics, Pohang, Gyeongbuk 790-784, Korea
}

\begin{abstract}
We consider the collinear ternary fission which is a sequential ternary decay with a very short time between the ruptures of two necks connecting the middle cluster of the ternary nuclear system and outer fragments. In particular, we consider the case where the Coulomb field of the first massive fragment separated during the first step of the fission produces a lower pre-scission barrier in the second step of the residual part of the ternary system. In this case, we obtain a probability of about $10^{-3}$ for the yield of massive clusters such as ${ }^{70} \mathrm{Ni},{ }^{80-82} \mathrm{Ge},{ }^{86} \mathrm{Se}$, and ${ }^{94} \mathrm{Kr}$ in the ternary fission of ${ }^{252} \mathrm{Cf}$. These products appear together with the clusters having mass numbers of $A=132-140$. The results show that the yield of a heavy cluster such as ${ }^{68-70} \mathrm{Ni}$ would be followed by a product of $A=138-148$ with a large probability as observed in the experimental data obtained with the FOBOS spectrometer at the Joint Institute for Nuclear Research. The third product is not observed. The landscape of the potential energy surface shows that the configuration of the $\mathrm{Ni}+\mathrm{Ca}+\mathrm{Sn}$ decay channel is lower about $12 \mathrm{MeV}$ than that of the $\mathrm{Ca}+\mathrm{Ni}+\mathrm{Sn}$ channel. This leads to the fact, that the yield of $\mathrm{Ni}$ and $\mathrm{Sn}$ is large. The analysis on the dependence of the velocity of the middle fragment on mass numbers of the outer products leads to the conclusion that, in the collinear tripartition channel of ${ }^{252} \mathrm{Cf}$, the middle cluster has a very small velocity, which does not allow it to be found in experiments.
\end{abstract}

PACS numbers: 21.60.Gx, 25.85.Ca

\section{INTRODUCTION}

True ternary fission of heavy nuclei, which has been discovered in the experiments recently, occurs with much smaller probability $\left(\sim 10^{-3}\right)$ compared to the binary fission [1 4]. These experimental studies of the decays in ${ }^{252} \mathrm{Cf}(\mathrm{sf}, \mathrm{fff})$ [1] and ${ }^{235} \mathrm{U}\left(\mathrm{n}_{\mathrm{th}}, \mathrm{fff}\right)$ [4] reactions with two fission fragment coincidences with two FOBOS-detectors [1] placed at $180^{\circ}$, using the missing mass approach, have established the phenomenon of collinear cluster tripartition (CCT). The third product is not observed. Only recently its dynamical properties could be investigated and it has been concluded that it proceeds collinearly. More details can be found, for example, in Refs. 5-7], and it is expected that the investigation of true ternary fission will allow us to extend our knowledge about fusion-fission processes. In the present work, we consider one of the dominant modes of the CCT.

Because of small probability, there are only a few experimental measurements of CCT as given in Refs. 1 [4]. Moreover, theoretical studies about ternary fission are very limited and some early works on this topic can be found, for example, in Refs. [5, [6, 8, 9]. In these early works it was found that the ternary fission process

\footnotetext{
* tashkhodjaev@inp.uz

† nasirov@jinr.ru

$\ddagger$ yohphy@knu.ac.kr
}

in heavy nuclei occurs preferably in collinear geometry, which was confirmed by the recent theoretical studies in Refs. [7, 10, 11]. In Ref. [7], the authors studied the difference between equatorial configurations and collinear configurations in ternary fission by calculating the potential energies for geometries of three fragments touching each other, i.e., a tri-nuclear system (TNS), where only the mass number of the central nucleus changes. On the other hand, the kinetic energies of the CCT products were evaluated in Ref. [10] to find that in most cases the velocity of the central fragment can be very small. This may be responsible for missing the third product in the experiments of Refs. [1 [4]. In Ref. [11] the CCT process has been considered as two sequential binary fissions. Namely, in the first stage the excited compound nucleus decays into two fragments in an asymmetric channel, then the heavier fragment decays further into two fragments. As a result, three fragments are obtained with comparable masses. In Ref. [11] only the yield of ternary fission fragments with comparable masses has been considered as it is similar to the case observed in the experiments performed by the FOBOS group [2]. The theoretical results of the yield of ${ }^{80} \mathrm{Ge}$ and ${ }^{84} \mathrm{Se}$ isotopes as the first step for the CCT products and the products ${ }^{70} \mathrm{Ni},{ }^{74,76} \mathrm{Zn}$, and ${ }^{82} \mathrm{Ge}$ in the second step in a sequential fission process are in good agreement with some of the corresponding experimental data on the mass distributions of the ${ }^{252} \mathrm{Cf}$ decay. This observation leads to the conclusion that these events can be associated with the sequential two step mechanism 


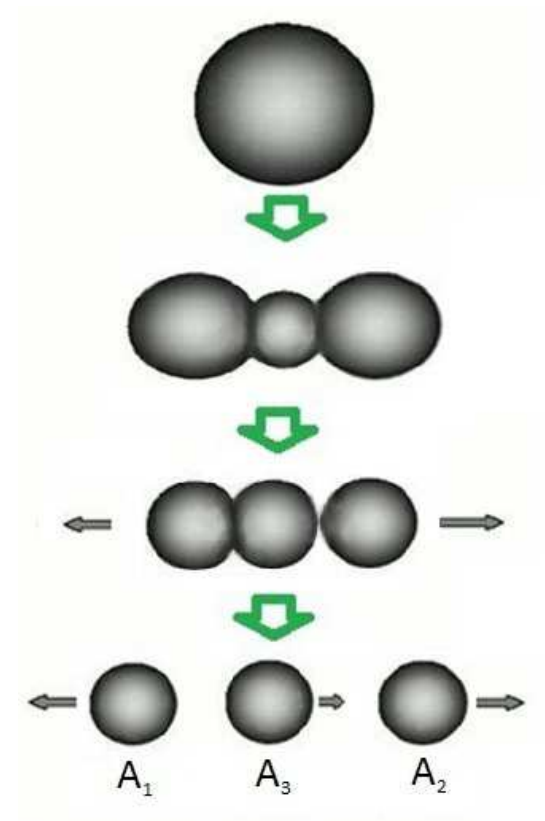

FIG. 1. (Color online) The CCT fission mechanism of a heavy nucleus in a sequential decay [1]. $A_{1}, A_{2}$, and $A_{3}$ are mass numbers of the fragments formed in the tri-nuclear system.

of CCT. However, the yields of ${ }^{70} \mathrm{Ni},{ }^{82} \mathrm{Ge}$, and ${ }^{84} \mathrm{Se}$ in coincidence with ternary fission masses $A=130-150$ observed in Ref. [4] with a large probability were not fully explained in Ref. [11].

Therefore, in the present paper we consider the mechanism of a sequential ternary fission with a very short time between the ruptures of the two necks connecting the middle cluster of the collinear TNS with its outer fragments. This mechanism is the almost-simultaneous ternary fission as illustrated in Fig. 1. The main goal of the present work is to pursue theoretical analysis of the ternary fission channels leading to the formation of the products of mass number $A=132-140$.

The collinear configuration of the tri-nuclear system undergoing fission is defined as follows. First, the three fragments are situated on one line and the border nuclei are numbered as " 1 " and "2", while the middle nucleus is labeled as "3" as shown in Fig. 2. Consequently, there is no nuclear interaction between the outer fragments "1" and "2". However, the Coulomb interaction between them is taken into account because of its longrange property. In fact, it was found to have a nontrivial role in the decay of TNS. The pre-scission barrier between fragments " 1 " and " 3 " decreases due to the Coulomb field of the fragment "2". For example, in the case of the sequential ternary fission of ${ }^{236} \mathrm{U}$, when ${ }^{132} \mathrm{Sn}$ forms as the fragment " 2 ", the pre-scission barrier between the fragments "2" and "3" is smaller than the one between the fragments "1" and "3" [1] . Certainly the massive fragment "2" is separated at the first step, then occurs the rupture of the second neck between fragments "1" and "3". We will discuss the probability of the rupture at the

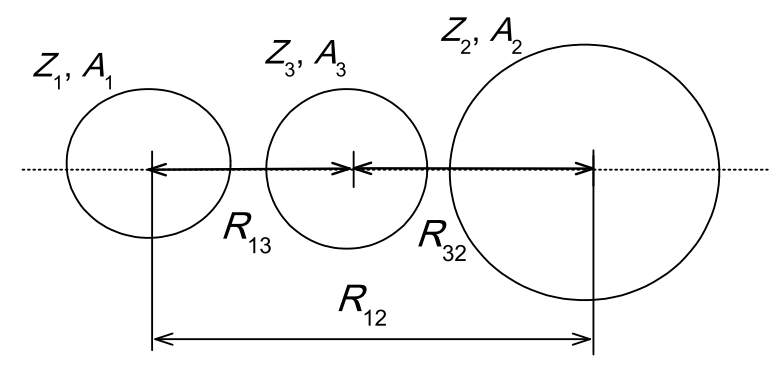

FIG. 2. The variables of the tri-nuclear system used in the analysis of the interaction energy between its fragments. Here, $Z_{i}$ is the charge number of fragment $i(i=1,2,3)$ and $R_{i j}$ is the distance between the mass centers of fragments $i$ and $j$.

second neck between "1" and "3", which decreases with increasing the distance $R_{32}$ that induces the decrease of the Coulomb field of the massive fragment "2". The definitions of the variables of TNS used in this analysis can be found in Fig. 2 .

\section{THEORETICAL APPROACH: FROM A DINUCLEAR SYSTEM TO A TRI-NUCLEAR SYSTEM}

In order to explore the mechanisms of the CCT process, we apply the theoretical framework of the dinuclear system (DNS) model 12 15]. In the present work, we estimate the total energy of the interacting system by calculate the sum of the binding energies of its constituents and the interaction potential energy between them. The minima of the potential energy surfaces (PES) are found by the variation of the charge and mass numbers of two fragments out of the three fragments and the distances between them. The PES is the two dimensional driving potential which depends on the charge numbers of two fragments of the collinear TNS. The distances $R_{13}$ and $R_{32}$ between centers of mass of fragments are found from the minimum value of the nucleus-nucleus interaction.

The fission process is considered as a formation of the elongated mononucleus (for example, superdeformed shape) which breaks down into two fragments as in the case of binary fission. The formation of the third cluster in the neck region and the splitting of this system into three fragments are related to the shape of the system such as hyperdeformation. Furthermore, the assumption of the formation of a heavier nucleus as the third fragment between the two main fission products is also introduced. 


\section{A. Total potential energy of a tri-nuclear system}

The study on the landscape of the potential energy surface (PES) is carried out to find minima and valleys, since, at local maxima, one can expect increased yields of the mass and charge distributions in the TNS undergoing fission process. It should be noted that the stage of transition from compound nucleus to the TNS configuration is not analyzed. Instead, we assume that TNS is formed during fission of the compound nucleus into a binary system. This process can occur in the sense of energy conservation. We refer to Ref. [16] for the hyperdeformed ${ }^{236} \mathrm{U}$ nucleus.

The PES is calculated as

$$
\begin{aligned}
& U\left(Z_{1}, A_{1}, \beta^{(1)}, Z_{2}, A_{2}, \beta^{(2)}, Z_{3}, A_{3}, \beta^{(3)}, R_{13}, R_{32}\right) \\
= & V_{\text {int }}\left(Z_{1}, A_{1}, \beta^{(1)}, Z_{2}, A_{2}, \beta^{(2)}, Z_{3}, A_{3}, \beta^{(3)}, R_{13}, R_{32}\right) \\
& +Q_{\operatorname{ggg}}\left(Z_{1}, A_{1}, Z_{3}, A_{3}\right),
\end{aligned}
$$

where $Z_{i}$ and $A_{i}$ are the charge number and mass number of the $i^{\text {th }}$ fragment of the TNS $(i=1,2,3)$, respectively, and $R_{i j}$ is the distance between the mass-centers of the $i^{\text {th }}$ and $j^{\text {th }}$ fragments. Here, $\beta^{(i)}=\left\{\beta_{2}^{(i)}, \beta_{3}^{(i)}\right\}$ is a set of deformation parameters of the fragment $i$, where $\beta_{2}^{(i)}$ and $\beta_{3}^{(i)}$ represent the quadrupole and octupole parts, respectively. The interaction potential $V_{\text {int }}$ between the fragments of TNS can be written as

$$
\begin{aligned}
& V_{\mathrm{int}}\left(Z_{1}, A_{1}, \beta^{(1)}, \beta^{(2)}, Z_{3}, A_{3}, \beta^{(3)}, R_{13}, R_{32}\right) \\
= & \sum_{i<j}^{3} V_{i j}\left(Z_{i}, A_{i}, \beta^{(i)}, Z_{j}, A_{j}, \beta^{(j)} ; R_{i j}\right),
\end{aligned}
$$

where $V_{i j}$ is the two-body interaction potential between the nuclei " $i$ " and " $j$ ". It contains two parts, namely, the nuclear part $V_{\text {nuc }}^{i j}$ and the Coulomb part $V_{C}^{i j}$, so that

$$
\begin{aligned}
& V_{i j}\left(Z_{i}, A_{i}, \beta^{(i)}, Z_{j}, A_{j}, \beta^{(j)} ; R_{i j}\right) \\
= & V_{\text {nuc }}^{i j}\left(Z_{i}, A_{i}, \beta^{(i)}, Z_{j}, A_{j}, \beta^{(j)} ; R_{i j}\right) \\
& +V_{C}^{i j}\left(Z_{i}, A_{i}, \beta^{(i)}, Z_{j}, A_{j}, \beta^{(j)} ; R_{i j}\right) .
\end{aligned}
$$

It is clear that $V_{\text {nuc }}^{12}=0$ since the fragments " 1 " and " 2 " are separated by the fragment " 3 " and, therefore, there is no overlap of their nucleon densities. The nuclear part of the nucleus-nucleus interaction $V_{\text {nuc }}^{i j}$ is calculated by using the double folding procedure 11], and the Coulomb part $V_{C}^{i j}$ is estimated by the Wong expression [17].

In Eq. (1), $Q_{\text {ggg }}$ is the reaction balance energy in ternary fission, which is written as

$$
\begin{aligned}
& Q_{\mathrm{ggg}}\left(Z_{1}, Z_{3}, A_{1}, A_{3}\right) \\
= & B_{1}\left(Z_{1}, A_{1}\right)+B_{2}\left(Z_{2}, A_{2}\right)+B_{3}\left(Z_{3}, A_{3}\right) \\
& -B_{C N}\left(Z_{C N}, A_{C N}\right) .
\end{aligned}
$$

The values of binding energies $B_{i}$ for ground states are taken from Refs. [18, 19].
In order to calculate the mass and charge distributions of the TNS in the pre-scission state, the minima and valleys of the PES are determined by computing the interaction potential $V_{\text {int }}$ as a function of $\left(Z_{1}, A_{1}, Z_{3}, A_{3}, R_{13}, R_{32}\right)$ since $\left(Z_{2}, A_{2}\right)$ can be defined through $\left(Z_{1}, A_{1}, Z_{3}, A_{3}\right)$ and $R_{12}=R_{13}+R_{32}$. This is done by taking $V_{\text {int }}$ as a function of $R_{13}$ and $R_{32}$ for each configuration of $\left\{Z_{1}, A_{1} ; Z_{3}, A_{3} ; Z_{2}, A_{2}\right\}$. (See Fig. 2 for the geometry.)

In order to find the dominant cluster states of PES, the charge (and mass) numbers of the two fragments are varied in the range of $2<Z_{1}<Z_{\mathrm{CN}} / 2$ and $2<Z_{3}<Z_{\mathrm{CN}} / 2$ $\left[A_{1, \min }<A_{1}\left(Z_{1}\right)<A_{1, \max }\right.$ and $A_{3, \min }<A_{3}\left(Z_{3}\right)<$ $\left.A_{3, \max }\right]$. The charge and mass numbers of the third fragment can be found from the corresponding conservation laws for them. The distances $R_{13}$ and $R_{32}$ between interacting nuclei are then varied to find $R_{13}^{(\min )}$ and $R_{32}^{(\min )}$ that correspond to the minimum values of the potential wells $V_{13}$ and $V_{32}$, respectively. It should be noted again that the potentials are affected by the Coulomb interaction $V_{12}^{C}$ of the border fragments.

This process allows us to find the mass number $A_{i}$ that corresponds to the minimum value of the PES for a given value of $Z_{i}$. For example, the value of $A_{1}$ can be found by minimizing the PES for each value of $A_{3}$ at fixed values of $Z_{1}$ and $Z_{3}$. From the set of the results calculated for PES as a function of $\left(Z_{1}, A_{1}, Z_{3}, A_{3}, R_{13}, R_{32}\right)$ we can establish the driving potential demonstrating the configurations of the TNS with the well-pronounced cluster states having closed shells. The three-dimensional driving potential $U_{\mathrm{dr}}\left(Z_{1}, A_{1} ; Z_{3}, A_{3}\right)$ is determined by the values of the PES in Eq. (11) corresponding to the minimum values of the potential wells in the nucleus-nucleus interaction $V_{\text {int }}$ between neighbor fragments as a function of the distances between their centers-of-mass:

$$
\begin{aligned}
& U_{\mathrm{dr}}\left(Z_{1}, A_{1}, \beta^{(1)}, \beta^{(2)}, Z_{3}, A_{3}, \beta^{(3)}\right) \\
= & U\left(Z_{1}, A_{1}, \beta^{(1)}, \beta^{(2)}, Z_{3}, A_{3}, \beta^{(3)}, R_{13}^{(\min )}, R_{32}^{(\min )}\right) .(5)
\end{aligned}
$$

A change of $A_{i}$ leads to the change of $Q_{\text {ggg }}$ which depends on the binding energies $B_{i}$. As a result, $U_{\mathrm{dr}}$ is sensitive to the mass distribution between the TNS fragments.

\section{B. Probability of the yield of ternary fission fragments}

The mass and charge distributions of the TNS fragments are related to the driving potential $U_{\mathrm{dr}}$. Therefore, the knowledge of $U_{\mathrm{dr}}$ allows us to calculate the yield of the products of ternary fission as in Ref. [11]:

$$
\begin{aligned}
Y\left(Z_{1}, A_{1} ; Z_{3}, A_{3}\right)= & P\left(Z_{1}, A_{1} ; Z_{3}, A_{3}\right) \\
& \times W_{13}\left(Z_{1}, A_{1} ; Z_{3}, A_{3}\right) \\
& \times W_{32}\left(Z_{1}, A_{1} ; Z_{3}, A_{3}\right),
\end{aligned}
$$

where $P\left(Z_{1}, A_{1} ; Z_{3}, A_{3}\right)$ is the probability of the charge and mass distributions of the TNS fragments. The probability of the formation of a TNS, $P\left(Z_{1}, A_{1} ; Z_{3}, A_{3}\right)$, can 
be found from the condition of a statistical equilibrium as in Ref. 20], i.e., the TNS has an equilibrium state before scission:

$$
P\left(Z_{1}, A_{1} ; Z_{3}, A_{3}\right)=P_{0} \exp \left[-U_{\mathrm{dr}}\left(Z_{1}, A_{1} ; Z_{3}, A_{3}\right) / T_{\mathrm{TNS}}\left(Z_{1}, A_{1} ; Z_{3}, A_{3}\right)\right],
$$

where $T_{\mathrm{TNS}}$ is the effective temperature of the TNS and $U_{\mathrm{dr}}\left(Z_{1}, A_{1} ; Z_{3}, A_{3}\right)$ is determined by the formula (5). The normalization coefficient for the yield probability is represented by $P_{0}$.

In Eq. (6), $W_{13}$ and $W_{32}$ are the decay probabilities of the TNS that are caused by overcoming the pre-scission barriers $B_{13}$ and $B_{32}$ which correspond to the separation of the first and second nuclei, respectively. Their explicit expressions can be found as [11]

$$
\begin{aligned}
& W_{13}\left(Z_{1}, A_{1} ; Z_{3}, A_{3}\right)=W_{13}^{0} \exp \left[-\frac{B_{13}}{T_{13}}\right], \\
& W_{32}\left(Z_{2}, A_{2} ; Z_{3}, A_{3}\right)=W_{32}^{0} \exp \left[-\frac{B_{32}}{T_{32}}\right],
\end{aligned}
$$

where $\left(B_{13}, B_{32}\right)$ and $\left(T_{13}, T_{32}\right)$ are the pre-scission barriers and the effective temperatures on these barriers the corresponding parts of the TNS. The barriers $B_{13}$ and $B_{32}$ prevent the separation of the outer fragments from the middle one. These pre-scission barriers are defined by the depth of the nucleus-nucleus potential well between neighbor fragments of the TNS. Here, $W_{13}^{0}$ and $W_{32}^{0}$ are normalization coefficients for the corresponding probability distributions.

The effective temperatures are determined by the excitation energy of the TNS which is generated by the descent of the system from the saddle point in binary fission. We assume that the third cluster is formed between the two parts of fissioning nuclei before their splitting. In this case, $E_{\text {TNS }}^{*}\left(Z_{1}, A_{1}, Z_{3}, A_{3}\right)$, the excitation energy of the system, is determined by the difference between the values at the saddle point and at the point of the minimum driving potential with the considered charge and mass numbers of clusters:

$$
E_{\mathrm{TNS}}^{*}\left(Z_{1}, A_{1}, Z_{3}, A_{3}\right)=E_{\mathrm{CN}}^{*}-U_{\mathrm{dr}}\left(Z_{1}, A_{1} ; Z_{3}, A_{3}\right) .
$$

The effective temperatures of the TNS, necks "1-3" and "2-3" are defined by the excitation energies on the minimum of the driving potential and pre-scission barriers $B_{13}$ and $B_{32}$, respectively:

$$
\begin{aligned}
T_{\mathrm{TNS}} & =\sqrt{\frac{12 E_{\mathrm{TNS}}^{*}}{A_{C N}}}, \\
T_{13} & =\sqrt{\frac{12 E_{13}^{*}}{\left(A_{1}+A_{3}\right)}}, \\
T_{32} & =\sqrt{\frac{12 E_{32}^{*}}{\left(A_{2}+A_{3}\right)}},
\end{aligned}
$$

where $E_{13}^{*}$ and $E_{32}^{*}$ are the excitation energies on the top of the pre-scission barrier the DNS "1-3" and of the DNS "2-3", respectively. These excitation energies are the result of sharing the TNS excitation energy between different degrees of freedom. The parts of $E_{\text {TNS }}^{*}$ corresponding to the decay axes $R_{13}$ and $R_{32}$ are estimated by assumption that their inertia masses are $A_{13}=A_{1}\left(A_{2}+A_{3}\right) / A_{\mathrm{CN}}$ and $A_{32}=A_{2}\left(A_{1}+A_{3}\right) / A_{\mathrm{CN}}$, respectively. Then the values of $E_{13}^{*}$ and $E_{32}^{*}$ are found from the effective temperature of the TNS:

$$
\begin{aligned}
& E_{13}^{*}\left(Z_{1}, A_{1}, Z_{3}, A_{3}\right)=\frac{T_{\mathrm{TNS}}^{2} A_{13}}{12}-B_{13}, \\
& E_{32}^{*}\left(Z_{1}, A_{1}, Z_{3}, A_{3}\right)=\frac{T_{\mathrm{TNS}}^{2} A_{32}}{12}-B_{32} .
\end{aligned}
$$

If the residual part of the TNS excitation energy $E_{\text {res }}^{*}=$ $E_{\mathrm{TNS}}^{*}-E_{13}^{*}-E_{32}^{*}$ is larger than the energy $B_{n}$ for the emission of neutrons from the TNS fragments, the ternary fission is accompanied by neutrons.

\section{INVESTIGATION OF TRIPARTITION IN THE ${ }^{252} \mathrm{Cf}(\mathrm{sf})$ REACTION}

\section{A. Potential energy surface showing the cluster formation in TNS}

In the experiment reported in Ref. [4], the ternary products were formed in the spontaneous fission of ${ }^{252} \mathrm{Cf}$ and the yields of ${ }^{68} \mathrm{Ni},{ }^{80-82} \mathrm{Ge},{ }^{94} \mathrm{Kr},{ }^{128,132} \mathrm{Sn}$, and ${ }^{144} \mathrm{Ba}$ were obtained. In the plot of the mass-mass distribution of two products (third one is missing) given in Fig. 10 of Ref. [4], these events were found to form a rectangle, and the authors of Ref. [4] assumed that the points in the right half of the rectangle likely reflect the shell effects around $N=88$.

The effect of the shell structure of the proton and neutron single-particle states in the formation of a tri-nuclear system and in its decay into the observed fission products is obviously seen in the mass-mass distribution data of Ref. [4]. This observation stimulates us to calculate PES, i.e., $V\left(Z_{1}, Z_{3}, A_{1}, A_{3}, R_{13}, R_{32}\right)$, and the driving potential $U_{\mathrm{dr}}\left(Z_{1}, A_{1}, Z_{3}, A_{3}\right)$ for the intermediate system preceding to their formation. The products of a CCT-decay should be formed before being separated from the other part of the system.

Our results for the PES are presented in Fig. 3 Each point in the driving potential $U_{\mathrm{dr}}\left(Z_{1}, A_{1}, Z_{3}, A_{3}\right)$ for the 


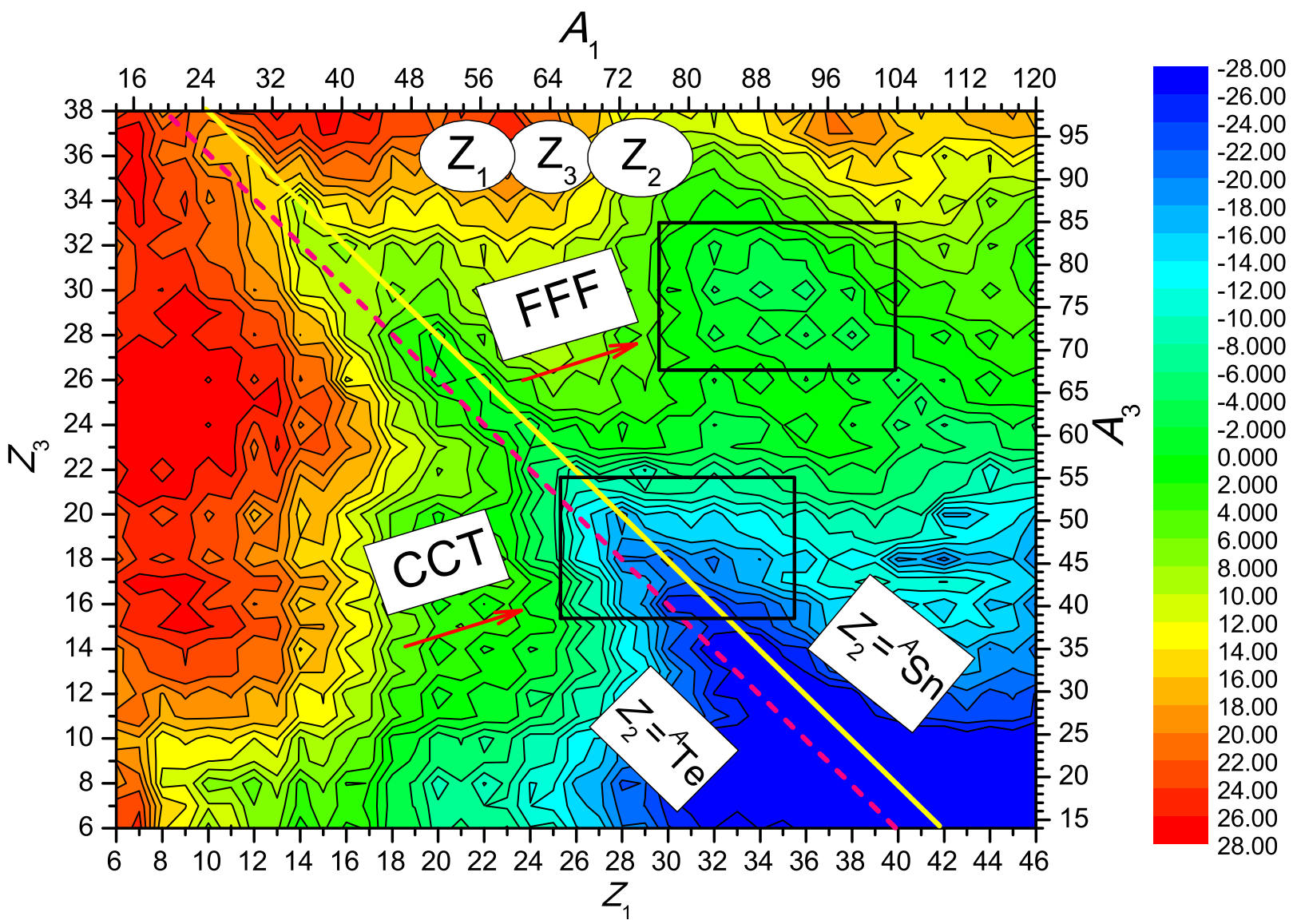

FIG. 3. (Color online) The potential energy surface of the ${ }^{252} \mathrm{Cf}(\mathrm{sf}, \mathrm{fff})$ reaction. The rectangle "CCT" shows the area of the mass numbers $Z_{1}\left(A_{1}\right)$ and $Z_{3}\left(A_{3}\right)$ which corresponds to the CCT products. The rectangle "FFF" shows the area of formation three fragments with the close mass numbers. The solid and dashed lines show the TNS configuration having ${ }^{132} \mathrm{Sn}$ and ${ }^{134} \mathrm{Te}$, respectively, as the outer nucleus $Z_{2}$.

TNS corresponds to a specific configuration (channel), which consists of three interacting nuclei placed in one line as shown in Fig. 2. In calculation of PES, the distances between the fragments are fixed at the values corresponding to the minimum values of the corresponding wells in the interaction potential between them (see Figs. (4) and (5). The quadrupole deformation parameters of the first-excited $2^{+}$state of nuclei [21] are used in calculation of PES.

The rectangle "CCT" shows the area of the mass numbers $Z_{1}\left(A_{1}\right)$ and $Z_{3}\left(A_{3}\right)$ which corresponds to the CCT products. The rectangle "FFF" shows the area of formation three fragments with the close mass numbers. The solid and dashed lines show the TNS configuration having ${ }^{132} \mathrm{Sn}$ and ${ }^{134} \mathrm{Te}$, respectively, as the outer nucleus $Z_{2}$. So, we can see the valley, which is the energetically minimum area $\left(Z_{2}=50\right.$ and $\left.Z_{2}=52\right)$ and corresponds to the ${ }^{252} \mathrm{Cf} \rightarrow \mathrm{f}_{1}+\mathrm{f}_{3}+{ }^{132} \mathrm{Sn}$ fission channel. The valley extends up to the area about $Z_{3}=28$. As was mentioned earlier, $Z_{3}$ is the charge number of the middle cluster. The phase space of the configurations corresponding to the blue color region is large. Therefore, the probability of finding the TNS of configurations with a lower potential energy is larger. The configuration of $\mathrm{Ni}+\mathrm{Ca}+\mathrm{Sn}$ has large probability compared with the $\mathrm{Ca}+\mathrm{Ni}+\mathrm{Sn}$ configuration since the PES value of the latter configuration is about $12 \mathrm{MeV}$ higher than that of the former configuration.

The calculations were performed to find the yield of the CCT products from the collinear geometry based on the formula of Eq. (6). In these calculations we found that the value of the pre-scission barrier plays the decisive role. Therefore, in the next section we discuss the behavior of the barriers $B_{13}$ and $B_{32}$ for the CCT channel of the $\mathrm{Ni}+\mathrm{Ca}+\mathrm{Sn}$ configuration.

\section{B. The decrease of the pre-scission barrier due to the Coulomb field of outer fragments}

The mechanism of almost sequential ruptures of the two necks connecting the fragments of a collinear TNS is suggested to explain the observed yields of heavy clusters such as $\mathrm{Ni}, \mathrm{Ge}$, and $\mathrm{Se}$ isotopes that appear with 


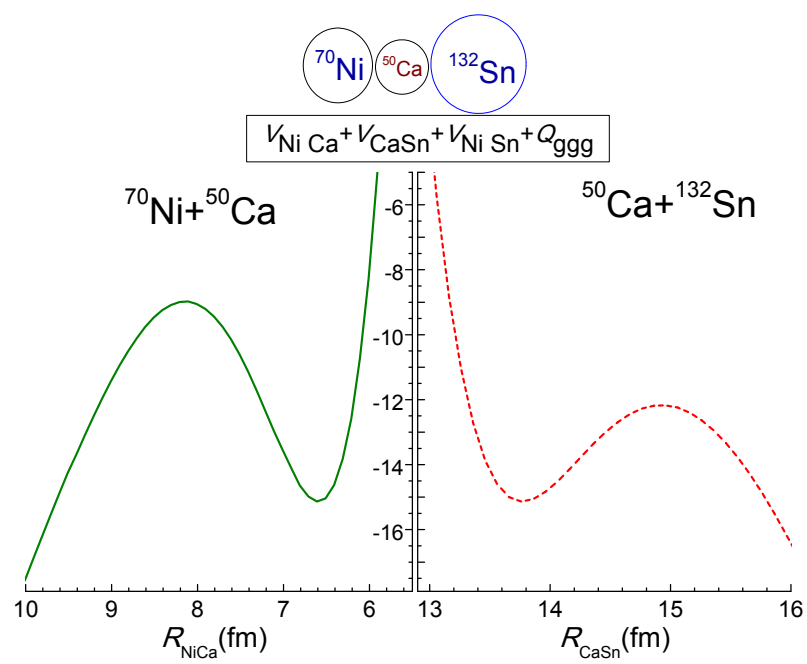

FIG. 4. (Color online) The pre-scission barriers $B_{\mathrm{Ni} \text { Ca }}$ and $B_{\mathrm{CaSn}}$ keeping TNS fragments together.

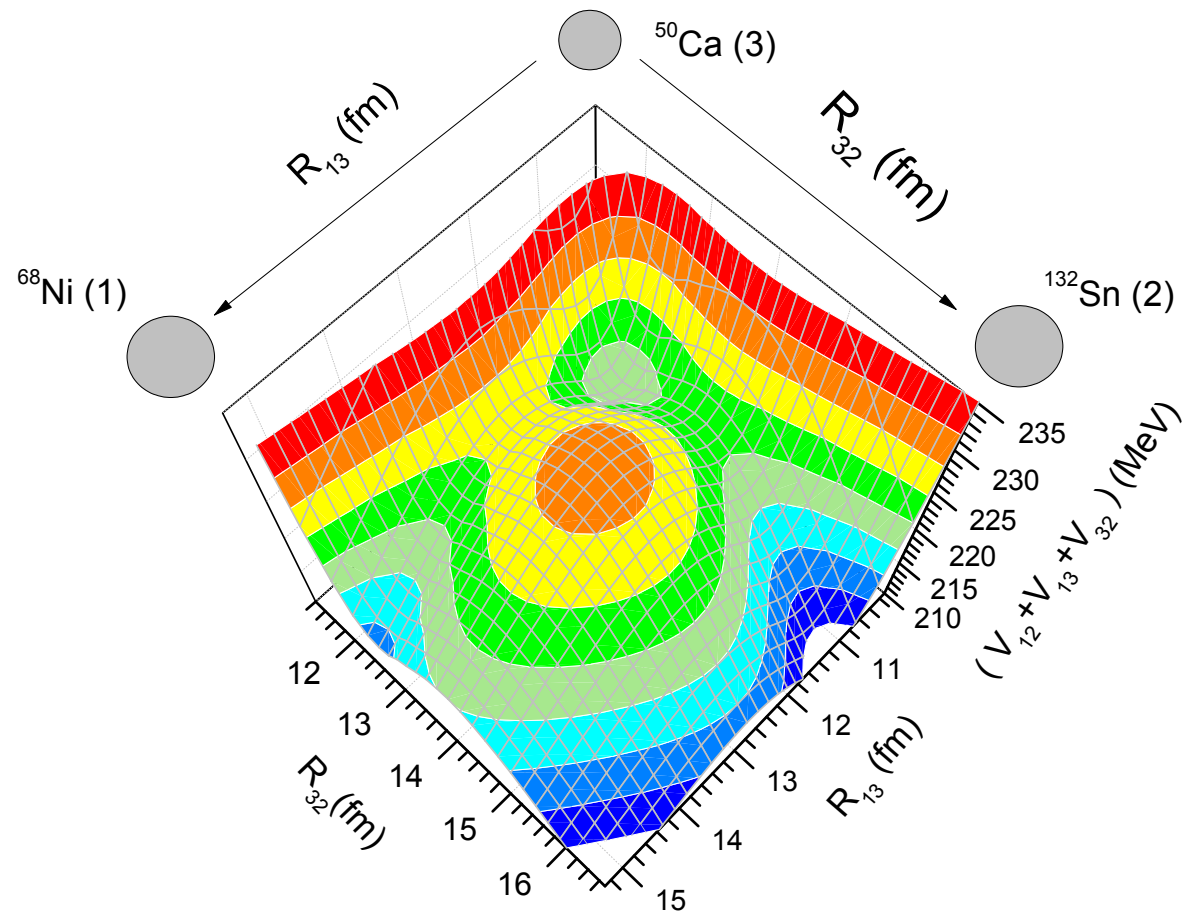

FIG. 5. (Color online) The total nucleus-nucleus interaction potential $V_{\text {int }}$ as a function of inter-center distances $R_{13}$ and $R_{32}$ between fragments of the TNS with collinear geometry.

the product having a mass number of $A=138-148$ in the CCT of ${ }^{252} \mathrm{Cf}[1,4]$. The PES shows the structure of valleys and local minima that correspond to the formation of heavy clusters observed in experiments as shown in Fig. 3. These fragments of a TNS should be emitted from the potential wells, and, therefore, it is important to estimate the depths of the potential wells, since heavy clusters are allowed to exist during a definite long time. In Fig. 4 the potential wells calculated for the TNS of $\mathrm{Ni}+\mathrm{Ca}+\mathrm{Sn}$, which forms a linear chain, are presented as functions of the distances between centers of the middle nucleus ( $\mathrm{Ca}$ ) and the outer nuclei ( $\mathrm{Ni}$ and $\mathrm{Sn}$ ). The values of these nucleus-nucleus potentials are shifted by the values of $Q_{\text {ggg }}$ as the contour plot of the PES [see 


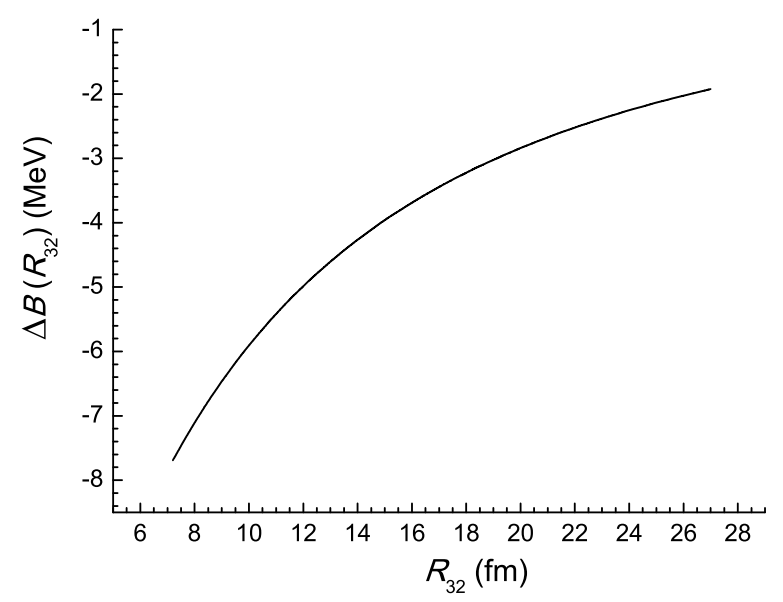

FIG. 6. The dependence of the pre-scission barrier $B_{13}$ for the decay of the binary system $\mathrm{Ni}+\mathrm{Ca}$ on the distance $R_{32}$ due to the Coulomb interaction of the massive third fragment $\mathrm{Sn}$ in the collinear geometry.

Fig. 3 Eq. (11)] to take into account the change of the intrinsic energy of the TNS.

For the calculation of the interaction potential $V_{\mathrm{CaSn}}$, the distance $R_{\mathrm{NiCa}}$ is fixed to the value corresponding to the minimum of $V_{\mathrm{NiCa}}$, while $V_{\mathrm{NiCa}}$ potential is calculated at the fixed value of $R_{\mathrm{CaSn}}$ that gives the minimum of $V_{\mathrm{CaSn}}$. The results for the nucleus-nucleus interaction between the nuclei of the collinear TNS of $\mathrm{Ni}+\mathrm{Ca}+\mathrm{Sn}$ as a function of the independent variables $R_{13}\left(R_{\mathrm{NiCa}}\right)$ and $R_{32}\left(R_{\mathrm{CaSn}}\right)$ are given by a three dimensional plot of the PES in Fig. 5 . The contour lines of the PES presented in Fig. 3 is calculated with the minimum value of the nucleus-nucleus interaction at $R_{13}=11 \mathrm{fm}$ and $R_{32}=12 \mathrm{fm}$ in Fig. 5. The decay of the TNS occurs due to the motion of the system along $R_{13}$ or $R_{32}$. The height of the pre-scission barrier is smaller in the direction along $R_{32}$ ( $R_{\mathrm{CaSn}}$ in Fig. 4) and, therefore, the massive fragment $\mathrm{Sn}$ is separated first from the TNS. This result is obtained by the use of Eqs. (8) and (9). If the residual $\mathrm{Ni}+\mathrm{Ca}$ part of the TNS does not decay, the binary decay would be observed since the $\mathrm{Ni}+\mathrm{Ca}$ system is considered as a superdeformed shape of ${ }^{48} \mathrm{Cd}$.

The excitation energy of the residual $\mathrm{Ni}+\mathrm{Ca}$ system should be large enough so that it decays into $\mathrm{Ni}$ and $\mathrm{Ca}$, if these nuclei are observed as CCT products. The probability of this event strongly depends on the position of the separated massive product, i.e., the Sn nucleus. The depth of the potential $V_{\mathrm{NiSn}}$, which is the pre-scission barrier $B_{\mathrm{NiSn}}$, changes as a function of the distance $R_{32}$. To show this phenomenon we estimate the change of the $B_{13}$ barrier, which is the difference between the maximum (on the barrier) and the minimum values of $V_{\text {int }}$ as a function of $R_{13}$ in Eq. (2). The dependence of the change of the barrier $B_{13}$ by the distance $R_{32}$ is reduced to a simple form of

$$
\Delta B_{13}\left(R_{32}\right)=\frac{Z_{1} Z_{2} e^{2}}{\left(R_{13}^{(B)}+R_{32}\right)}-\frac{Z_{1} Z_{2} e^{2}}{\left(R_{13}^{(\min )}+R_{32}\right)},
$$

where $e^{2}=1.44 \mathrm{MeV} \cdot \mathrm{fm}$. The dependence of $B_{13}$ on $R_{32}$ is presented in Fig. 6. The negative values mean the decrease of the depth of the potential well $\left(B_{13}\left(R_{32} \rightarrow\right.\right.$ $\left.\infty)+\Delta B_{13}\left(R_{32}\right)\right)$ in the interaction of the $\mathrm{Ni}+$ Ca system. The main observation of the present work is that the presence of the third fragment is important to cause the decay of the $\mathrm{Ni}+\mathrm{Ca}$ system in an easier way. The presence of the third massive fragment Sn makes the prescission barrier shallower by $4 \mathrm{MeV}$, and thus the decay probability of the $\mathrm{Ni}+\mathrm{Ca}$ system increases.

By taking into account the change of the pre-scission barrier one can obtain reasonable results for the yields of the $\mathrm{Ni}$ isotopes followed by the emission of massive $\mathrm{Sn}$ isotopes from the formula in Eq. (6) that includes the effects of the pre-scission barriers $B_{13}$ and $B_{32}$. The results are presented in Figs. 7 and 8 In the former figure we use $Z_{1}\left(A_{1}\right)$ and $Z_{2}\left(A_{2}\right)$ axes for the plot, while in the latter figure we use $Z_{1}\left(A_{1}\right)$ and $Z_{3}\left(A_{3}\right)$ axes.

Although the calculated yields of heavy clusters such as $\mathrm{Ni}, \mathrm{Ge}$, and $\mathrm{Se}$ isotopes are found to be in good agreement with the experimental data, there still remains a difference between the mass numbers of the massive CCT products of ${ }^{252} \mathrm{Cf}$ observed in Refs. 11, 4], namely $A=138-148$ which overlap with our results with $A=132-140$ presented in Figs. 7 and 8 . The strong yield of the products with mass numbers $A=132$ 140 indicates the important role of the magic number of neutrons at $N=82$. This difference may be ascribed to that we use the tabulated masses of Refs. [18, 19] to obtain the binding energies of nuclei. This procedure, in fact, gives the binding energies of the ground states, but we may have deformed nuclei at the scission point, which is highly probable for massive nuclei. But we should remind the procedure of calculation of the PES by variation of the charge and mass numbers of the two fragments of TNS $\left(2<Z_{1}<Z_{\mathrm{CN}} / 2\right.$ and $2<Z_{3}<Z_{\mathrm{CN}} / 2\left[A_{1, \min }<A_{1}\left(Z_{1}\right)<A_{1, \max }\right.$ and $\left.\left.A_{3, \text { min }}<A_{3}\left(Z_{3}\right)<A_{3, \max }\right]\right)$. The depends of shell corrections on the deformation should be studied for the most of the numerous (some thousands) combinations. Since the primary goal of this work is to demonstrate the possibility of the formation of the $\mathrm{Ni}, \mathrm{Ge}$, and $\mathrm{Se}$ isotopes and their yields in the CCT mechanism, we leave more accurate and sophisticated description of the production of massive isotopes of $A=138-148$ to our future studies.

\section{ESTIMATION OF THE KINETIC ENERGY OF THE MIDDLE FRAGMENT}

The range of the kinetic energy of the middle fragment "3" can be estimated by applying the energy and momentum conservation laws. For simplicity, we assume that 


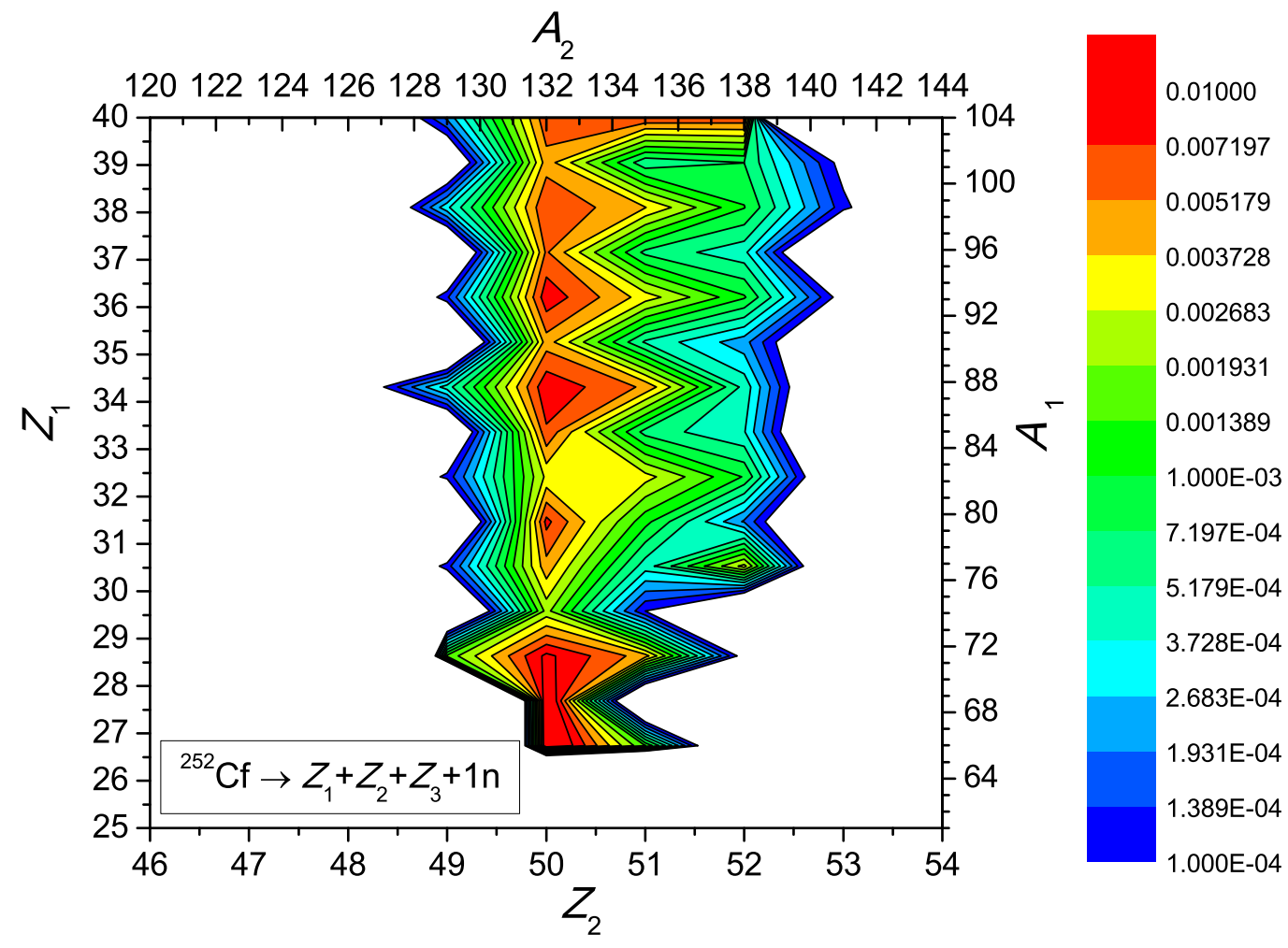

FIG. 7. (Color online) Theoretical results for the yield of the outer fragments ${ }^{A_{1}} Z_{1}$ and ${ }^{A_{2}} Z_{2}$ of the TNS formed at the spontaneous fission of ${ }^{252} \mathrm{Cf}$.

the kinetic energy of the binary process is determined by the Coulomb barrier of the nucleus-nucleus interaction. The first step of the sequential collinear ternary fission is the separation of the right fragment " 2 " as shown in Fig. 1. The sum of the kinetic energies of this fragment and the residual part of the TNS is the same as the Coulomb repulsion between them, which leads to

$$
\begin{aligned}
\frac{Z_{1} Z_{2} e^{2}}{R_{13}+R_{23}+d}+\frac{Z_{3} Z_{2} e^{2}}{R_{23}+d} & =\frac{m\left(A_{1}+A_{3}\right) v_{13}^{2}}{2} \\
& +\frac{m A_{2} v_{2}^{2}}{2} \\
m\left(A_{1}+A_{3}\right) v_{13}+m A_{2} v_{2} & =0
\end{aligned}
$$

where $v_{13}$ and $v_{2}$ are the relative velocities of the DNS "13" and of the separated fragment "2", respectively, in the laboratory frame. The free parameter $d$ is introduced to decrease the sum of the total Coulomb barriers that can not be larger than the reaction energy balance $Q_{\text {ggg }}$ given in Eq. (4). The second step of the sequential collinear ternary fission is a decay of the DNS "13" into two fragments " 1 " and " 3 ". The sum of their kinetic energies is then equal to the Coulomb repulsion between them so that

$$
\begin{aligned}
& \frac{Z_{1} Z_{3} e^{2}}{R_{13}}=\frac{m A_{1} v_{1}^{\prime 2}}{2}+\frac{m A_{3} v_{3}^{\prime 2}}{2}, \\
& m A_{1} v_{1}^{\prime}+m A_{3} v_{3}^{\prime}=0,
\end{aligned}
$$

where $v_{1}^{\prime}$ and $v_{3}^{\prime}$ are the velocities of the fragments " 1 " and " 3 ", respectively, in the moving frame with velocity $v_{13}$ in the opposite direction to $v_{2}$. Therefore, we have

$$
\begin{aligned}
& v_{1}=v_{1}^{\prime}+v_{13}, \\
& v_{3}=-v_{3}^{\prime}+v_{13} .
\end{aligned}
$$

We then obtain $v_{3}$ as a function of the mass numbers of the outer fragments ${ }^{\mathrm{A}_{1}} \mathrm{Z}_{1}$ and ${ }^{\mathrm{A}_{2}} \mathrm{Z}_{2}$ and the results are presented in Fig. 9. The negative values of $v_{3}$ mean that its direction is opposite to the direction of $v_{2}$. This 


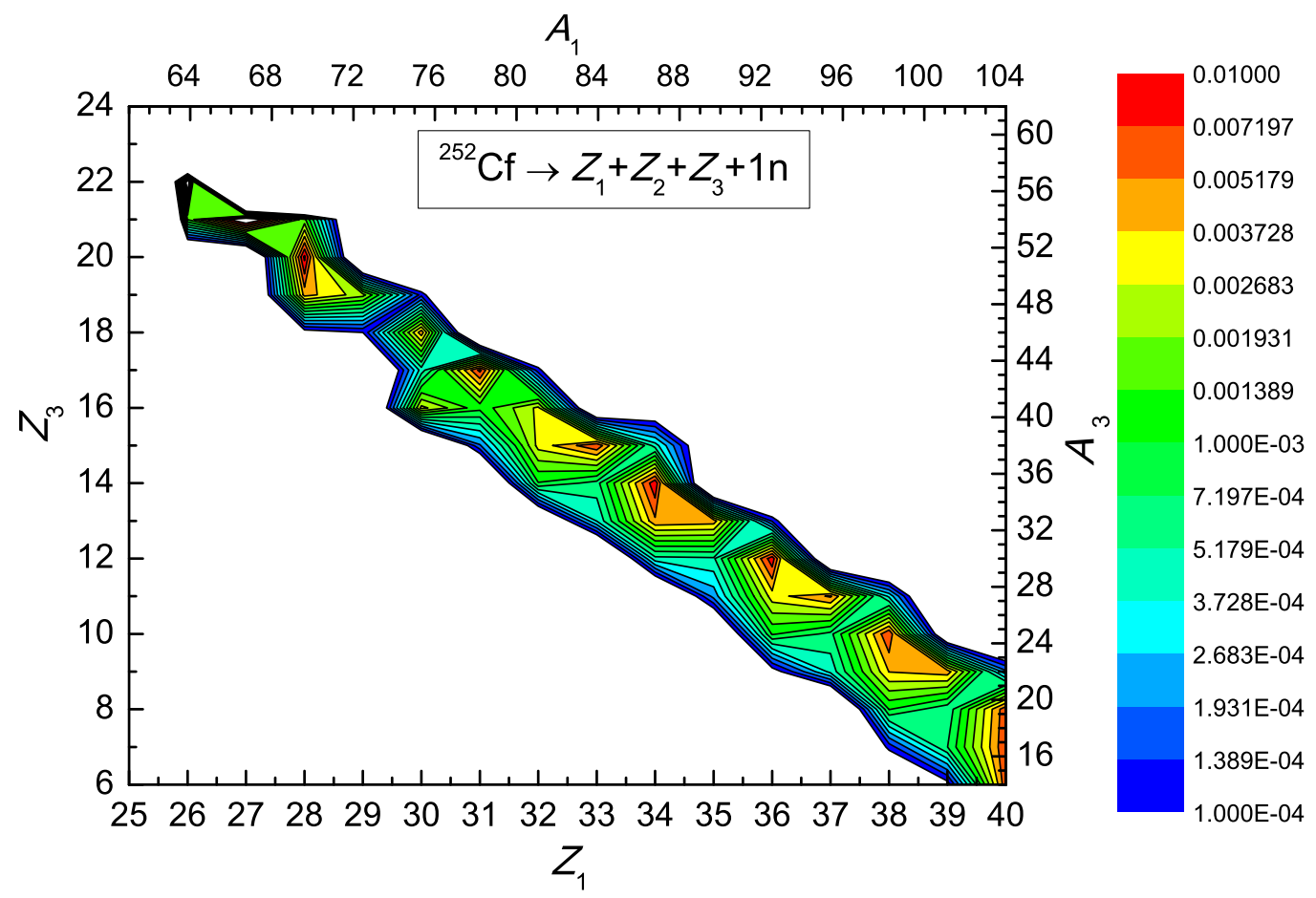

FIG. 8. (Color online) Theoretical results for the yield of the outer ${ }^{A_{1}} Z_{1}$ and middle ${ }^{A_{3}} Z_{3}$ fragments of the TNS formed at the spontaneous fission of ${ }^{252} \mathrm{Cf}$.

figure also allows us to find the region of mass numbers $A_{1}$ and $A_{2}$ where the velocity of the middle cluster is large enough to be registered in experiments. One of the puzzles in the experimental data on the collinear tripartition presented in Refs. [1, 2, 4] is the missing of the third fragment. As can be understood from this analysis, the main physical reason of this phenomenon is the smallness of the velocity of the "missing" third product.

In Fig. 9one can see that the third product has a small velocity $\left(\left|v_{3}\right|<0.25 \mathrm{~cm} / \mathrm{ns}\right)$ for the case of $A_{1}=60$ 80 and $A_{3}=24-64$, which means that the range of mass numbers for the massive fragment is $A_{2}=108$ 168. This region overlaps with the observed mass region, where a Ni-like product with a mass number of $A_{1}=68-72$ was observed with a massive product of $A_{2}=136-144$ [1, 2, 4]. In the case of the symmetric masses, $A_{1} \sim A_{2} \sim A_{3}$, we have a small velocity of the middle fragment $A_{3}$, namely, we get $v_{3}=0.3-0.4 \mathrm{~cm} / \mathrm{ns}$. The range of the mass numbers where the third middle fragment has an observable velocity is found to be $A_{1}=100-120$ and $A_{3}=4-16$ (i.e., $A_{2}=116-148$ ) which corresponds to the well-known ternary fission with emission of the light nuclei with a mass number of $A_{3}=4-$ 12 [22, 23]. In the experiments reported in Refs. 22, 23], all the three ternary fission products were registered. The other range of mass numbers of the outer fragments of TNS which allows to the observation of the middle fragment is $A_{1}=104-112$ and $A_{3}=64-90\left(A_{2}=50-84\right)$. The decay channel of $A_{2}<100$ has a very small probability to be realized since the pre-scission barrier $B_{32}$ is sufficiently high. Our analysis on the sequential true ternary fission shows that the possibility of observing the middle fragment in this case is small.

\section{CONCLUSION}

In this work, we suggested a sequential ternary fission process with a very short time between the ruptures of two necks connecting the middle cluster of the collinear tri-nuclear system. The necessity of this mechanism is revealed in the decrease of the pre-scission barrier of the 


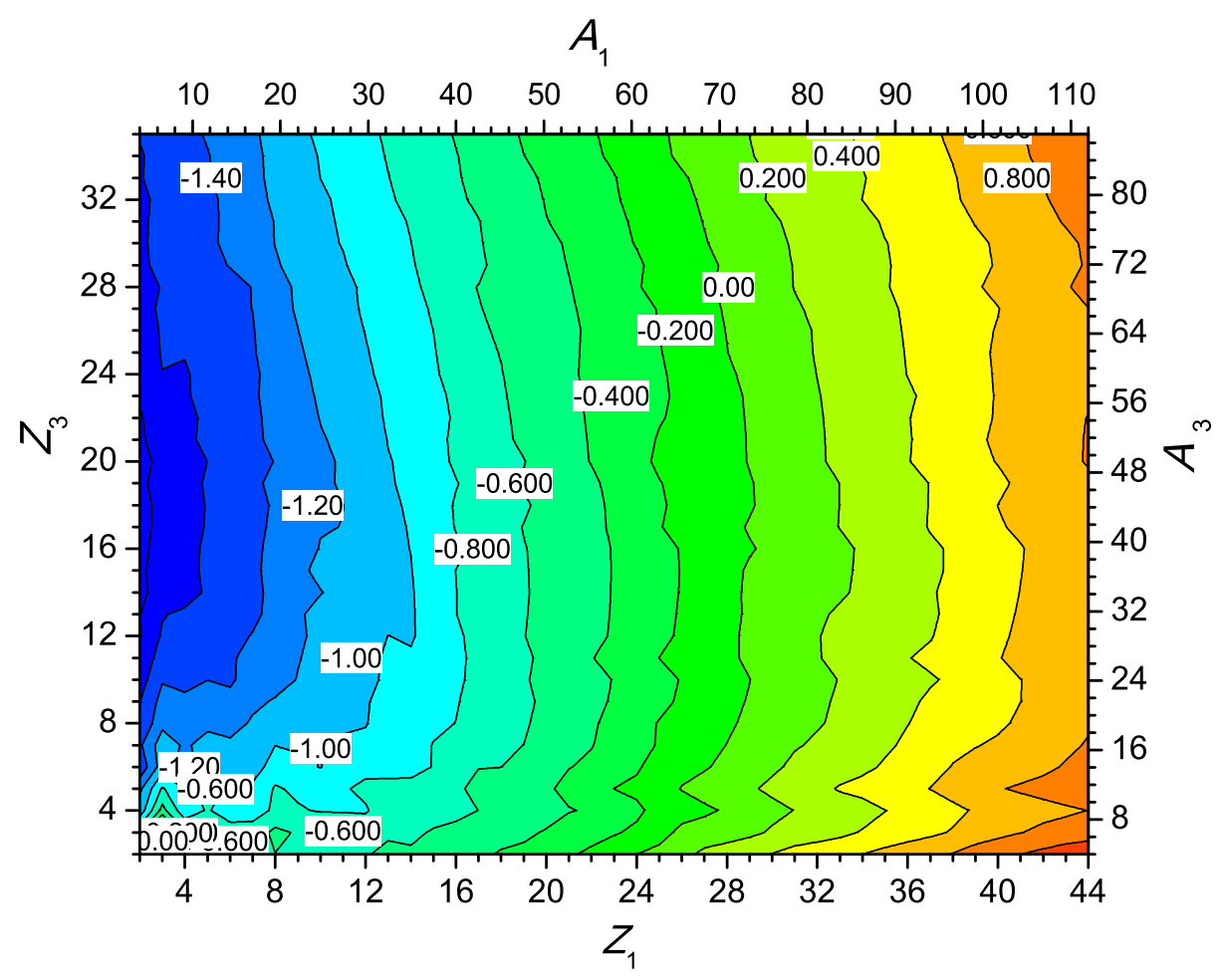

FIG. 9. (Color online) The contour map of the calculated velocity $v_{3}$ (in $\mathrm{cm} / \mathrm{ns}$ ) of the middle ${ }^{\mathrm{A}_{3}} \mathrm{Z}_{3}$ fragment of the TNS formed at the spontaneous fission of ${ }^{252} \mathrm{Cf}$ as a function of the mass numbers of the outer fragments ${ }^{\mathrm{A}_{1}} \mathrm{Z}_{1}$ and ${ }^{\mathrm{A}_{2}} \mathrm{Z}_{2}$. The negative values of $v_{3}$ mean that its direction is opposite to the direction of $v_{2}$.

residual part of the TNS due to the Coulomb field of the massive fragment being separated first. This mechanism leads to the probability of about $10^{-3}$ for the yield of massive clusters such as ${ }^{70} \mathrm{Ni},{ }^{80-82} \mathrm{Ge},{ }^{86} \mathrm{Se}$, and ${ }^{94} \mathrm{Kr}$ produced with a product of $A=132-140$ in the CCT of ${ }^{252} \mathrm{Cf}$. The yields of such products were observed in coincidence with a massive product of $A=138-148$ with a relatively large probability in the experiments of the FOBOS group at the FLNR of JINR (Dubna).

To verify the realization of this mechanism, the total potential energy of the chain-like TNS was calculated as a sum of $Q_{\text {ggg }}$ and the nucleus-nucleus interaction potential energy between its constituents. The minima and valleys of the PES related to the shell effects in nuclei were determined by using the binding energies obtained from the well-known mass tables of Refs. [18, 19] and the calculation of the interaction potential for the charge and mass numbers of the three fragments as a function of distances between their centers-of-mass. The distances $R_{13}$ and $R_{32}$ between interacting nuclei were varied to find the minimum values of the potential wells of $V_{13}$ and $V_{32}$, respectively, which are affected by the Coulomb interaction $V_{12}^{C}$ of the border fragments. The driving potential as a function of the charge and mass numbers of two fragments was obtained at the values of the distances $R_{13}^{(\mathrm{min})}$ and $R_{32}^{(\mathrm{min})}$ that correspond to the minimum values of the nucleus-nucleus interactions $V_{13}$ and $V_{32}$, respectively.
In order to find the dominant cluster states of the TNS, the driving potential $U_{\mathrm{dr}}\left(Z_{1}, A_{1}, Z_{3}, A_{3}\right)$ was calculated for the values of the charge (mass) numbers of the two fragments in the ranges of $2<Z_{1}<Z_{\mathrm{CN}} / 2$ and $2<Z_{3}<Z_{\mathrm{CN}} / 2\left[A_{1, \min }<A_{1}\left(Z_{1}\right)<A_{1, \max }\right.$ and the $\left.A_{3, \min }<A_{3}\left(Z_{3}\right)<A_{3, \max }\right]$. The analysis of the results allows us to find the mass number $A_{i}$ corresponding to the minimum value of the PES for a given value of $Z_{i}$. The calculated total potential energy as a function of $\left(Z_{1}, A_{1}, Z_{3}, A_{3}\right)$ enabled us to establish the three dimensional driving potential that demonstrates the configurations of TNS with probable cluster states in the pre-fission states.

Finally, the contour lines of the three dimensional driving potential showed the structure of a valley corresponding to the formation of the outer cluster with $Z_{2}=50$ or $N_{2}=82$ at the ternary fission, which corresponds to the fission channel of ${ }^{252} \mathrm{Cf} \rightarrow \mathrm{f}_{1}+\mathrm{f}_{3}+{ }^{132} \mathrm{Sn}$. It was found that the valley extends up to the area of about $Z_{3}=28$ and the probability of a configuration having lower potential energy for the TNS is large. Therefore, the configuration of $\mathrm{Ni}+\mathrm{Ca}+\mathrm{Sn}$ has a large probability in comparison with the configuration of $\mathrm{Ca}+\mathrm{Ni}+\mathrm{Sn}$ since the PES value of the latter configuration is about $12 \mathrm{MeV}$ higher than that of the former configuration.

The dependence of the velocity of the middle cluster on the mass numbers $A_{1}$ and $A_{2}$ was also analyzed for the case of the collinear tripartition of ${ }^{252} \mathrm{Cf}$. The main 
physical reason associated with the collinear tripartition is the smallness of the missed third product. We found that, in the range of the mass numbers $A_{1}=60-80$ and $A_{2}=132-140$, the middle fragment has a very small velocity, which is in agreement with the observed range of values presented in Refs. [1, 2, 4]. This means that it is indeed difficult to observe the middle product of a collinear tripartition of ${ }^{252} \mathrm{Cf}$ producing $\mathrm{Ni}$ with the second product having a mass number of $A_{2}=132-140$. In the case of the symmetric masses, $A_{1} \sim A_{2} \sim A_{3}$, we have a small velocity of the middle fragment $A_{3}$, namely, we get $v_{3}=0.3-0.4 \mathrm{~cm} / \mathrm{ns}$. The smallness of the middle cluster velocity may be a reason of its missing in the collinear tripartition in the ${ }^{252} \mathrm{Cf}(\mathrm{sf}, \mathrm{fff})$ [1] and ${ }^{235} \mathrm{U}\left(\mathrm{n}_{\mathrm{th}}, \mathrm{fff}\right)$ [4] reactions.

The mass ranges of the two outer products, where the middle fragment can be observed, are i) $A_{1}=100-120$ and $A_{2}=130-140$ which corresponds to the well-known ternary fission with an emission of the light nucleus with $A_{3}=4-12$ [22, 23] and ii) $A_{1}=90-110$ and $A_{2}=100-$ 132

\section{ACKNOWLEDGMENTS}

We thank our colleagues, Prof. D. V. Kamanin and Prof. Yu. V. Pyatkov, for fruitful discussions. A.K.N. is grateful to RFBR for partial support and W.v.O. thanks FLNR of JINR for their hospitality during his stay in Dubna. A.K.N. was supported in part by the MSIP of the Korean Government under the Brain Pool Program No. 142S-1-3-1034. The work of Y.O. was supported by the National Research Foundation of Korea under Grant No. NRF-2013R1A1A2A10007294.
[1] Yu. V. Pyatkov et al., Collinear cluster tri-partition of ${ }^{252} \mathrm{Cf}(\mathrm{sf})$ and in the ${ }^{235} \mathrm{U}\left(\mathrm{n}_{\mathrm{th}}, \mathrm{f}\right)$ reaction, Eur. Phys. J. A 45, 29 (2010).

[2] Yu. V. Pyatkov, D. V. Kamanin, Yu. N. Kopach, A. A. Alexandrov, I. A. Alexandrova, S. B. Borzakov, Yu. N. Voronov, V. E. Zhuchko, E. A. Kuznetsova, Ts. Panteleev, and A. N. Tyukavkin, Collinear cluster tripartition channel in the reaction ${ }^{235} \mathrm{U}\left(\mathrm{n}_{\mathrm{th}}, \mathrm{f}\right)$, Yad. Fiz. 73, 1350 (2010), [Phys. Atom. Nucl. 73, 1309 (2010)].

[3] Yu. V. Pyatkov, D. V. Kamanin, A. A. Alexandrov, I. A. Alexandrova, N. A. Kondratjev, E. A. Kuznetsova, N. Jacobs, V. Malaza, D. Phan Minh, and V. E. Zhuchko, Presumable scenario of one of the collinear cluster tripartition modes, Int. J. Mod. Phys. E 20, 1008 (2011).

[4] Yu. V. Pyatkov et al., The collinear cluster tri-partition (CCT) of ${ }^{252} \mathrm{Cf}(\mathrm{sf})$ : New aspects from neutron gated data, Eur. Phys. J. A 48, 94 (2012).

[5] H. Diehl and W. Greiner, Theory of ternary fission in the liquid drop model, Nucl. Phys. A229, 29 (1974).

[6] G. Royer, On the fission of ${ }^{56} \mathrm{Ni}$ and ${ }^{48} \mathrm{Cr}$ rotating nuclei, J. Phys. G 21, 249 (1995).

[7] K. Manimaran and M. Balasubramaniam, All possible ternary fragmentations of ${ }^{252} \mathrm{Cf}$ in collinear configuration, Phys. Rev. C 83, 034609 (2011).

[8] J. A. Maruhn and W. Greiner, Collective effects on mass asymmetry in fission, Phys. Rev. C 13, 2404 (1976).

[9] D. N. Poenaru, R. A. Gherghescu, and W. Greiner, Complex fission phenomena, Nucl. Phys. A747, 182 (2005).

[10] K. R. Vijayaraghavan, W. von Oertzen, and M. Balasubramaniam, Kinetic energies of cluster fragments in ternary fission of ${ }^{252} \mathrm{Cf}$, Eur. Phys. J. A 48, 27 (2012).

[11] R. B. Tashkhodjaev, A. K. Nasirov, and W. Scheid, Collinear cluster tripartition as sequential binary fission in the ${ }^{235} \mathrm{U}\left(\mathrm{n}_{\mathrm{th}}, \mathrm{f}\right)$ reaction, Eur. Phys. J. A 47, 136 (2011).

[12] N. V. Antonenko, E. A. Cherepanov, A. K. Nasirov, V. P. Permjakov, and V. V. Volkov, Competition between com- plete fusion and quasi-fission in reactions between massive nuclei. The fusion barrier, Phys. Lett. B 319, 425 (1993).

[13] N. V. Antonenko, E. A. Cherepanov, A. K. Nasirov, V. P. Permjakov, and V. V. Volkov, Compound nucleus formation in reactions between massive nuclei: Fusion barrier, Phys. Rev. C 51, 2635 (1995).

[14] V. V. Volkov, From deep inelastic transfer reactions to complete fusion of nuclei, Acta Phys. Pol. B 30, 1517 (1999).

[15] A. Nasirov, A. Fukushima, Y. Toyoshima, Y. Aritomo, A. Muminov, S. Kalandarov, and R. Utamuratov, The role of orientation of nucleus symmetry axis in fusion dynamics, Nucl. Phys. A759, 342 (2005).

[16] M. Csatlósa et al., Resonant tunneling through the triplehumped fission barrier of ${ }^{236} \mathrm{U}$, Phys. Lett. B 615, 175 (2005).

[17] C.-Y. Wong, Interaction barrier in charged-particle nuclear reactions, Phys. Rev. Lett. 31, 766 (1973).

[18] G. Audi, A. H. Wapstra, and C. Thibault, The Ame2003 atomic mass evaluation: (II). Tables, graphs and references, Nucl. Phys. A729, 337 (2003).

[19] P. Möller, J. R. Nix, W. D. Myers, and W. J. Swiatecki, Nuclear ground-state masses and deformations, At. Data Nucl. Data Tables 59, 185 (1995).

[20] L. G. Moretto and J. S. Sventek, A theoretical approach to the problem of partial equilibration in heavy ion reactions, Phys. Lett. B58, 26 (1975).

[21] S. Raman, C.W. Nestor, Jr., and P. Tikkanen, Transition probability from the ground to the first-excited $2^{+}$state of even-even nuclides, At. Data Nucl. Data Tables 78, 1 (2001).

[22] F. Gönnenwein, Ternary and quaternary fission, Nucl. Phys. A734, 213 (2004).

[23] F. Gönnenwein, M. Mutterer, and Y. Kopatch, Ternary and quaternary fission, Europhysics News 36, 11 (2005). 